(pp 200-206)

\title{
The Promotional Effect of Low-intensity Direct Current Stimulation with Electrode Placement of Negative Poles at Wound Site on Pressure Ulcer Healing
}

Yoshiyuki YOSHIKAWA, PT, MS

Day service Miyabinosato

Masaharu SUGIMOTO, PT, MS

Faculty of Rehabilitation, Kobegakuin University

Noriaki MAESHIGE, PT, OT, PhD

Department of Rehabilitation, Eikokai Doi Hospital

Noriaki MAESHIGE, PT, OT, PhD, Mikiko UEMURA, PT

Faculty of Rehabilitation, Kobe International University

Mikiko UEMURA, PT

Department of Rehabilitation, Yoshida Hospital

Atsushi TAKAO, PT, Kazuhiro MATSUDA, PT

Department of Rehabilitation, Nakayama Clinic

Hiroto TERASHI, MD, PhD

Department of Plastic and Reconstructive Surgery, Kobe University Graduate School of Medicine

Purpose: The purpose of this study is to evaluate the effects of low-intensity direct current (LIDC) with electrode placement of negative poles at wound site on pressure ulcer healing.

Subject: The subjects were elderly patients having a pressure ulcer (sacrum, 2 cases; thoracic supine, 2 cases; ilium, 1 case; greater trochanter, 1 case; lateral malleolus, 1 case), and healings of these ulcers had been retarded. The DESIGN-R of these ulcers were between 13 and 19 .

Methods: The negative electrode of silver chloride was inserted into the foam dressing on the wound surface, and the positive pole was attached on intact skin around the wound. Electrical stimulation (intensity: $80 \mu \mathrm{A}$; frequency: $2 \mathrm{~Hz}$; pulse length: $250 \mathrm{msec}$; duration: 40 minutes) were administrated five times per week. On every treatment, opposite electrodes were shunted by electrical cable after the stimulation.

Result: Size reductions were observed on all ulcers treated with LIDC and these ulcers were healed completely at $5 \sim 10$ weeks after the beginning of LIDC stimulation.

Conclusion: The pressure ulcer healings were promoted by negative LIDC stimulation. It is suggested that LIDC stimulation with consideration for electrical polarity could be effective for shortening of the pressure ulcer healing period. 